\title{
Neutrophil function in systemic lupus erythematosus and other collagen diseases
}

\author{
H. Al-hadithy, D. A. ISEnBERG, ${ }^{*}$ I. E. AdDison, A. H. Goldstone, \\ AND M. L. SNAITH†
}

From the Departments of Haematology and $\nmid$ Rheumatology, University College Hospital, London

SUMMARY Using a whole blood technique we assessed neutrophil migration, phagocytosis, and killing in a group of 20 patients with systemic lupus erythematosus (SLE) and in 8 patients with other connective tissue disorders. In the untreated cases of SLE neutrophil migration was significantly depressed, but it was usually normal in the treated group. This may be attributable either to an intrinsic neutrophil abnormality or to a humoral factor. Although isolated abnormalities of phagocytosis and killing were observed in SLE, these functions were normal when the patients were considered as a group. The treated patients with other collagen diseases showed enhanced migration in both autologous and control plasma, normal phagocytosis, and enhanced killing in autologous plasma only. The small group of untreated, non-SLE patients showed some depression of all 3 functions. There was no correlation between neutrophil function and clinical activity of disease. In the SLE patients there was no correlation between neutrophil function and circulating immune complexes.

Most reports of neutrophil functions in systemic lupus erythematosus (SLE) agree that abnormalities occur but disagree about their nature and aetiology. For example, Brandt and Hedberg ${ }^{1}$ and Notani and colleagues $^{2}$ found impaired phagocytosis, whereas Hällgren et al. ${ }^{3}$ found it to be increased. Similarly, while Zivkovic and Baum ${ }^{4}$ reported normal chemotaxis in SLE, Clark et al. ${ }^{5}$ showed that chemotaxis was defective, especially in a subset of their SLE patients who tended to be younger, with earlier onset of the disease and higher DNA binding.

Several previous authors ${ }^{2}{ }^{6-8}$ have postulated the existence of a serum inhibitor in SLE. Others, including Brandt and Hedberg ${ }^{1}$ and Landry ${ }^{9}$ have suggested that a cellular defect is responsible for neutrophil dysfunction.

Conflicting results and postulates also abound in the reports of neutrophil function in rheumatoid arthritis. Brandt and Hedberg ${ }^{1}$ and Hällgren et al. ${ }^{3}$ both found normal phagocytosis, but Corberand $e t$ al. ${ }^{10}$ and Wilton et al. ${ }^{11}$ noted impaired phagocytosis.

Accepted for publication 26 January 1981.

*D.A.I. is a Jules Thorn research fellow.

Correspondence to $\operatorname{Dr}$ A. H. Goldstone, Department of Haematology, University College Hospital, Gower Street, London WC1E 6JJ.
In the present paper, with the development of a highly reproducible whole blood technique to assess neutrophil migration, phagocytosis, and killing, ${ }^{12} 13$ we re-evaluate neutrophil function in SLE and other connective tissue disorders. Furthermore, an attempt was made to correlate abnormalities of neutrophil function with disease activity, with treatment, and with levels of circulating immune complexes, since the latter have been implicated in the pathogenesis of both SLE and rheumatoid arthritis. ${ }^{14} 15$

\section{Patients and methods}

SLE PATIENTS

Twenty patients with SLE were studied repeatedly, with the result that 71 samples were tested. Each fulfilled 4 or more of the American Rheumatism Association's criteria for the classification of the disease. ${ }^{16}$ Disease activity was classified as inactive, moderately active, or severely active at each of the consultations before the results of any investigations were available. Severe activity was judged to be present if 3 or more of the following features were present: pleuritis, pericarditis, arthralgia, myalgia, vasculitis, cerebral, or renal disease. Patients with 1 or 2 of these features were considered to have moderately active disease and to be 'inactive' when 
none of these problems was present. Treated patients received various combinations of prednisone, azathioprine, and/or levamisole. A few patients were on other medication such as nonsteroidal antiinflammatory drugs, but these were not separated in the analysis.

Patients with other collagen diseases. Eight patients, 5 with rheumatoid arthritis, 2 with Sjögren's syndrome, and 1 with scleroderma, were studied. These patients were studied repeatedly, with the result that 24 samples were tested. Disease activity was assessed clinically as inactive, moderately active, or severely active. Four patients were receiving treatment with levamisole and one with prednisolone.

\section{METHODS}

In brief, blood was mixed $1: 1$ with Hanks's balanced salt solution. Migration was measured by placing the diluted blood in plastic caps which were inverted on to $3 \mu \mathrm{m}$ micropore membranes resting on filter paper soaked in medium. After 2 hours' incubation at $37^{\circ} \mathrm{C}$ in a moisture chamber the membranes were rinsed with saline, fixed with propan-2-01, stained with haematoxylin, and cleared with cedar wood oil, so that migration could be measured microscopically by the leading front method. ${ }^{12}$

Phagocytosis and killing were assessed by mixing blood and Candida guilliermondii at a ratio of $2: 1$ candida : neutrophil and 60-minute incubation at $37^{\circ} \mathrm{C}$ with end-over-end rotation. Cytocentrifuge preparations were stained with May-GrünwaldGiemsa to estimate the number of candida per 100 polymorphonuclear leucocytes and the percentage showing the colour change indicating cell death. ${ }^{13}$

Haematological parameters. The haemoglobin and leucocyte counts were measured on a Coulter $\mathbf{S}$ counter and the neutrophil count derived by scoring the percentage neutrophils from a 100-cell differential count. The haemoglobin levels ranged from $5.0 \mathrm{~g} / \mathrm{dl}$ to $16 \cdot 3 \mathrm{~g} / \mathrm{dl}$ and the neutrophil count from 1.0 to $14 \cdot 1 \times 10^{9} / 1$.

Circulating immune complexes (CIC). The CIC levels were measured by a modification ${ }^{17}$ of the polyethylene glycol precipitation technique. ${ }^{18}$ The IgG content of the redissolved precipitate was assayed using a laser nephelometer (Hyland Laboratory, Thetford, Norfolk). The upper limit of normal established in 30 healthy volunteers is $100 \mu \mathrm{g} \mathrm{l}^{-1} \mathrm{IgG}$.

Statistical methods. Statistical analysis of our data was carried out with the help of the MRC Biostatistics Unit, Cambridge. Use was made of paired $t$ test and 2-sample $t$ test and, where appropriate, of analysis of variance and rank correlation techniques. The $1 \%$ level of significance was used throughout as a convenient guide to assessing the significance of departures from a null hypothesis.

\section{Results}

Normal controls. A total of 39 samples from healthy volunteers were employed in the neutrophil migration studies and 47 in the candida phagocytosis and killing experiments. In all instances the results fell within the previously established normal ranges. ${ }^{1213}$

Neutrophil function in SLE. When all the SLE patients were considered together (i.e., treated and untreated), no significant abnormality in neutrophil migration, phagocytosis, or killing was detected in either autologous or control plasma (Table 1). For all 3 neutrophil functions no significant differences were detected between tests performed in autologous and control plasma ( $\mathrm{p}$ always $>0 \cdot 1$ ).

However, in the untreated cases considered as a group (Fig. 1) neutrophil migration was significantly depressed; in $40 \%$ and $32 \%$ of samples (in autologous and control plasma respectively), migration $<80 \%$ of the normal control.

Although candida phagocytosis and killing were normal overall in the untreated group, around $20 \%$ of samples showed impaired uptake and killing ( $<80 \%$ the normal control). Among the samples showing reduced phagocytosis and killing, in approximately $50 \%$ phagocytosis and killing returned to the normal range when the tests were performed in control plasma.

Table 1 Neutrophil function (mean $\pm 1 S D$ ) in $S L E$

\begin{tabular}{|c|c|c|c|c|}
\hline \multirow{2}{*}{$\begin{array}{l}\text { Neutrophil } \\
\text { function }\end{array}$} & \multirow{2}{*}{$\begin{array}{l}\text { Plasma in which } \\
\text { test performed }\end{array}$} & \multicolumn{3}{|l|}{ Patients } \\
\hline & & $A l l(n=71)^{*}$ & Treated $(n=45)$ & Non-treated $(n=26)$ \\
\hline $\begin{array}{l}\text { Migration } \\
\text { Phagocytosis } \\
\text { Killing }\end{array}$ & $\begin{array}{l}\text { Autologous } \\
\text { Control } \\
\text { Autologous } \\
\text { Control } \\
\text { Autologous } \\
\text { Control }\end{array}$ & $\begin{array}{l}0.98 \pm 0.30(\mathrm{NS}) \dagger \\
0.98 \pm 0.25(\mathrm{NS}) \\
0.97 \pm 0.18(\mathrm{NS}) \\
0.93 \pm 0.16(\mathrm{NS}) \\
0.94 \pm 0.19(\mathrm{NS}) \\
0.93 \pm 0.18(\mathrm{NS})\end{array}$ & $\begin{array}{l}1.08 \pm 0.31 \text { (NS) } \\
1.04 \pm 0.25 \text { (NS) } \\
1.01 \pm 0.18 \text { (NS) } \\
0.93 \pm 0.175 \text { (NS) } \\
0.95 \pm 0.21 \text { (NS) } \\
0.92 \pm 0.19 \text { (NS) }\end{array}$ & $\begin{array}{c}0.8 \pm 0.21(\mathrm{P}<0.001) \\
0.87 \pm 0.21(\mathrm{P}<0.01) \\
0.91 \pm 0.14(\mathrm{NS}) \\
0.93 \pm 0.13(\mathrm{NS}) \\
0.93 \pm 0.14 \text { (NS) } \\
0.945 \pm 0.16(\mathrm{NS})\end{array}$ \\
\hline
\end{tabular}

* $\mathrm{n}=$ Number of samples tested (69, migration; 71, phagocytosis and killing).

$+\mathrm{NS}=$ No significant difference from normal control. 


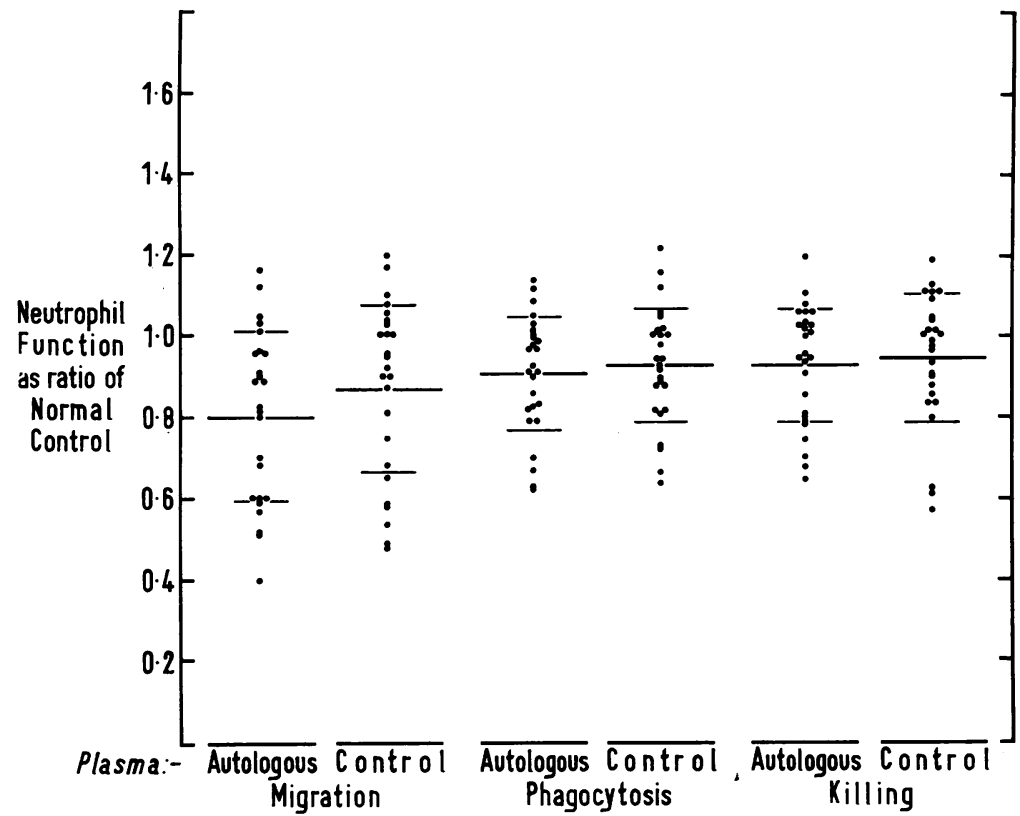

Fig. 1 Neutrophil migration, phagocytosis, and killing in the untreated group of SLE patients.

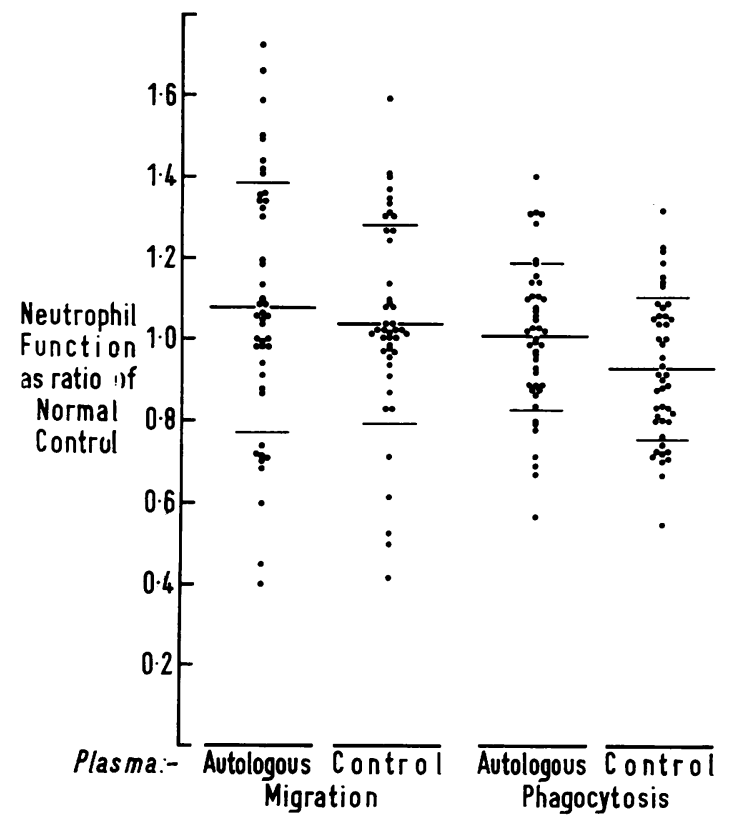

Relationship between neutrophil function and activity of SLE. There was no correlation between disease activity and neutrophil function.

Effect of treatment in SLE. When the treated patients were considered as a group, neutrophil migration was found to be normal. However, in 9 


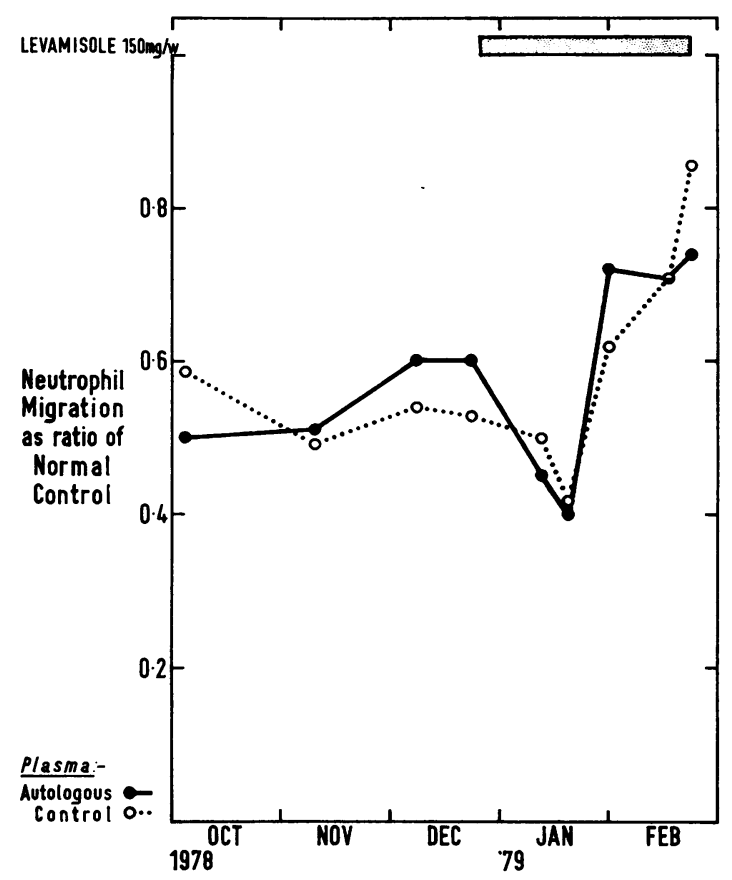

Fig. 3 Neutrophil migration in a treated (with levamisole) case of SLE, showing a lack of response to treatment when the test was performed in both autologous and control plasma. were normal overall whether the tests were performed in autologous or control plasma. However, in autologous plasma $13.3 \%$ and $28.9 \%$ of samples showed abnormal $(<80 \%)$ phagocytosis and killing respectively. The figures for control plasma were $22 \cdot 2 \%$ and $26 \cdot 7 \%$ respectively.

Circulating immune complexes in SLE. Twelve of the 20 SLE patients had raised CIC levels. There were no correlations between disease activity or neutrophil function tests and levels of CIC.

Neutrophil function in other collagen diseases. Neutrophil migration was significantly enhanced (Fig. 4) in both autologous and control plasma (mean $1 \cdot 18 \pm 0 \cdot 19$ and $1 \cdot 17 \pm 0 \cdot 15$ respectively; $p<0 \cdot 001$ ), but phagocytosis was normal $(1.02 \pm 0.19$ and $0.96 \pm 0.18$ in autologous and control plasma respectively). Killing was enhanced $(1 \cdot 18 \pm 0 \cdot 22$, $\mathrm{p}<0.005)$ in autologous plasma, but normal $(1.03 \pm 0 \cdot 16)$ in control plasma. There was no correlation between neutrophil function and disease activity.

Only 3 patients were not being treated at the time of the study. It was thus not possible to perform an adequate statistical analysis of the effect of treatment on neutrophil function in these disorders. However the results in the 6 samples obtained from the untreated patients showed the following results in autologous and control plasma respectively: mean

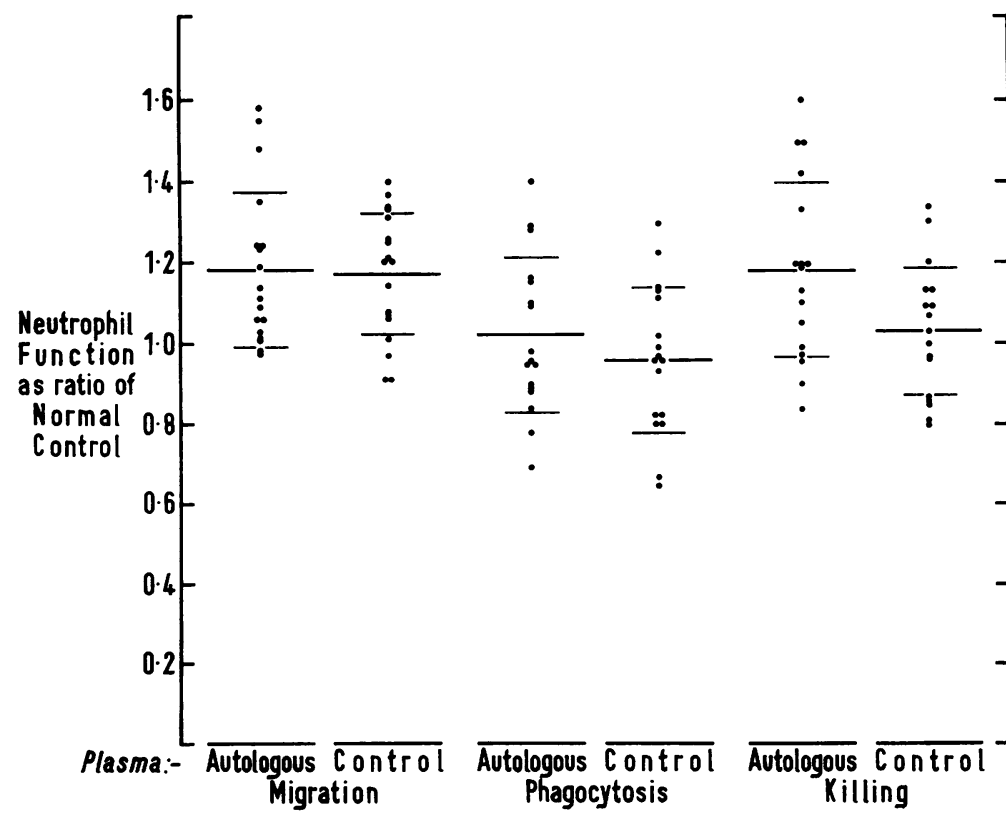

Fig. 4 Neutrophil migration, phagocytosis, and killing in the treated group of patients with other collagen diseases. 
neutrophil migration $0 \cdot 60 \pm 0 \cdot 29$ and $0 \cdot 72 \pm 0 \cdot 25$; mean candida phagocytosis $0.92 \pm 0.32$ and $0.91 \pm 0.28$; mean candida killing $0.90 \pm 0.20$ and $0 \cdot 82 \pm 0 \cdot 22$.

Haematological correlations. There were no correlations between the haemoglobin level and any of the neutrophil functions. However, there were correlations between the neutrophil count and migration in both autologous and control plasma $(\mathrm{r}=0.53, \mathrm{p}<0.001$, and $\mathrm{r}=0.49, \quad \mathrm{p}<0.001$ respectively). Neither phagocytosis nor killing in either autologous or control plasma could be correlated with the neutrophil count.

\section{Discussion}

In the present study it is clearly shown that neutrophil migration is consistently reduced in untreated SLE. The effect of treatment by various combinations of prednisolone, azathioprine, and levamisole was to normalise migration.

In approximately half the cases reduced migration was not corrected by autologous plasma. The present study therefore provides evidence of both an intrinsic neutrophil defect and of a serum inhibitor of neutrophil migration. Our study is in accord with most previous work, which has shown reduced neutrophil migration in SLE $^{5-9}$ but reconciles studies which have demonstrated either the existence of a serum inhibitor ${ }^{78}$ or of an intrinsic neutrophil defect. ${ }^{9}$

Although, overall, there were no statistically significant differences in candida phagocytosis or killing compared with normal controls, in either autologous or control plasma, some patients did show marked defects in these tests.

Previous authors have reported both overall decreased ${ }^{1269}$ and increased ${ }^{3}$ phagocytosis in SLE, and killing has received little previous attention. The reason for the discrepancy between the present study and that of others is not clear, but it may relate to the difference types of SLE patients studied and to the different techniques used.

No correlation of neutrophil function was found in the SLE patients with clinical states. This lack of correlation agrees with the observations by Zurier, ${ }^{6}$ who studied particle uptake by neutrophils and lysosomal enzyme release.

The present study did not confirm the association of decreased neutrophil phagocytosis in SLE with neutropenia. ${ }^{1}$ In contrast, neutropenia was significantly associated with impaired neutrophil migration.

Like some authors, ${ }^{19}$ though unlike others, ${ }^{20}$ we found no relationship between CIC levels and either neutrophil function or disease activity.

In the treated nonlupus group neutrophil migration was enhanced in both autologous and control plasma. This supports an earlier study on rheumatoid arthritis by Jacobs and Goetzal ${ }^{21}$ and is in accord with the stimulation of chemotaxis by levamisole as previously noted in normal volunteers ${ }^{22}$ and in patients with rheumatoid arthritis. ${ }^{23}$ In our treated nonlupus patients neutrophil phagocytosis was normal. In contrast previous authors ${ }^{101124}$ have shown reduced phagocytosis in rheumatoid arthritis; this discrepancy may be the result of the different drug therapy our patients received.

In conclusion our results indicate that neutrophil function is frequently abnormal in SLE and other collagen diseases and that it is often improved by therapy. However, the precise significance of such neutrophil defects in the pathogenesis of collagen disease remains obscure.

We thank Dr John Cawley for his helpful advice in the preparation of the manuscript, Dr John Fletcher for providing the candida used in phagocytosis and killing, and Miss Paula Richardson for secretarial assistance. Dr David Isenberg gratefully acknowledges the support of the Jules Thorn Foundation. We thank Dr John Morrow for measuring the circulating immune complex levels.

\section{References}

1 Brandt L, Hedberg H. Impaired phagocytosis by peripheral blood granulocytes in systemic lupus erythematosus. Scand J Haematol 1969; 6: 348-53.

2 Notani G W, Kenyon A J, Zurier R B. Altered neutrophil function induced by serum from patients with systemic lupus erythematosus. Adv Exp Med Biol 1976; 73: 147-54.

3 Hällgren R, Håkansson L, Venge P. Kinetic studies of phagocytosis. 1. The serum independent particle uptake by PMN from patients with rheumatoid arthritis and systemic lupus erythematosus. Arthritis Rheum 1978; 21: 107-13.

4 Zivkovic M, Baum J. Chemotaxis of polymorphonuclear leukocytes from patients with systemic lupus erythematosus and Felty's syndrome. Immunol Commun 1972; 1: $39-49$.

5 Clark R A, Kimball H R, Decker J L. Neutrophil chemotaxis in systemic lupus erythematosus. Ann Rheum Dis 1974; 33: 167-72.

6 Zurier R B. Reduction of phagocytosis and lysosomal enzyme release from human leucocytes by serum from patients with systemic lupus erythematosus. Arthritis Rheum 1976; 19: 73-8.

7 Alvarez I, Vazquez J J, Fontan G, Gil A, Barbado J, Ojeda $J$ A. Neutrophil chemotaxis and serum chemotactic activity in systemic lupus erythematosus. Scand $J$ Rheumatol 1978; 7: 69-74.

8 Perez H D, Lipton M, Goldstein I M. A specific inhibitor of complement (C5) derived chemotactic activity in serum from patients with systemic lupus erythematosus. $J$ Clin Invest $1978 ; 62: 29-38$.

9 Landry M. Phagocyte function and cell-mediated immunity in systemic lupus erythematosus. Arch Dermatol 1977; 113: 147-54. 
10 Corberand J, Amigues H, de Larrard B, Pradere J. Neutrophil function in rheumatoid arthritis. Scand $J$ Rheumatol 1977; 6: 49-52.

11 Wilton J M A, Gibson T, Chuck C M. Defective phagocytosis by synovial fluid and blood polymorphonuclear leucocytes in patients with rheumatoid arthritis. 1. The nature of the defect. Rheumatol Rehabil 1978; suppl; 25-36.

12 Al-Hadithy H, Addison I E, Goldstone A H. Use of whole blood in the measurement of neutrophil migration. $J$ Clin Pathol 1981; 34: 158-62.

13 Al-Hadithy H, Addison I E, Goldstone A H. A rapid whole-blood technique for assessment of neutrophil phagocytosis and killing. Clin Lab Haematol 1981; 3: 85-8.

14 Okuda K, Okamoto R, Noguchi Y, Tadokoro I. An investigation of leukocyte function and phagocytosis of immune complexes in patients with rheumatoid arthritis. Jpn J Exp Med 1975; 45: 1-17.

15 Cats A, Lafeber G J M, Klein F. Immunoglobulin phagocytosis by granulocytes from sera and synovial fluids in various rheumatoid and non-rheumatoid diseases. Ann Rheum Dis 1975; 34: 146-55.

16 Cohen A S, Reynolds W E, Franklin E C, et al. Preliminary criteria for the classification of systemic lupus erythematosus. Bull Rheum Dis 1971; 21: 643-8.

17 Poulton T A, Crowther M E, Hay F C, Nineham L J. Immune complexes in ovarian cancer. Lancet 1978; ii: 72-3.
18 Creighton W D, Lambert P M, Miescher P A. Detection of antibodies and soluble antigen-antibody complexes by precipitation with polyethylene glycol. J Immunol 1973; 111: 1219-27.

19 Inman R D, Fong J K K, Pussel B A, Hughes G R V. The Clq binding assay in SLE: discordance with disease activity. Ann Rheum Dis 1980; 39: 193.

20 Abrass C K, Nies K M, Louie J S, Border W A, Glassock R J. Correlation and predictive accuracy of circulating immune complexes with disease activity in patients with systemic lupus erythematosus. Arthritis Rheum 1980; 23: 273-82.

21 Jacobs J C, Goetzl E J. 'Streaking leucocyte factor,' arthritis and pyoderma gangrenosum. Pediatrics 1975; 56: $570-8$.

22 Anderson R, Oosthuizen R, Theron A, van Rensburg A J. The in vitro evaluation of certain neutrophil and lymphocyte functions following the ingestion of $150 \mathrm{mg}$ oral dose of levamisole: assessment of the extent and duration of stimulation of neutrophil chemotaxis, protein iodination and lymphocyte transformation. Clin Exp Immunol 1979; 35 : 478-83.

23 Mowat A G. Neutrophil chemotaxis in rheumatoid arthritis. Effect of D-penicillamine, gold salts and Levamisole. Ann Rheum Dis 1978; 37: 1-8.

24 Turner R A, Schumacher H R, Myers A R. Phagocytic function of polymorphonuclear leukocytes in rheumatic diseases. J Clin Invest 1973; 52 : 1632-5. 